\title{
Sterol regulatory element-binding proteins involved in reprogramming of lipid droplet formation after rotavirus infection
}

\author{
Ahsan Naveed ${ }^{1 \dagger}$, Yeong-Bin Baek ${ }^{1 \dagger}$, Mahmoud Soliman ${ }^{1,2 \dagger}$, Muhammad Sharif ${ }^{1}$, Sang-lk Park ${ }^{1 *}$, Mun-II Kang ${ }^{1 *}$ \\ ${ }^{1}$ Laboratory of Veterinary Pathology, College of Veterinary Medicine, Chonnam National University, Gwangju 61186, Korea \\ ${ }^{2}$ Department of Pathology \& Clinical Pathology, Faculty of Veterinary Medicine, Assiut University, Assiut 71526, Egypt
}

Received October 7, 2021

Accepted December 20, 2021

Corresponding author:

Sang-Ik Park

E-mail: sipark@jnu.ac.kr

https://orcid.org/0000-0003-1709-0324

Corresponding author:

Mun-Il Kang

E-mail: mikang@jnu.ac.kr

https://orcid.org/0000-0003-1595-1717

${ }^{\dagger}$ These first three authors contributed

equally to this work.

\begin{abstract}
Species A rotaviruses (RVAs) replicate and assemble their immature particles within electron dense compartments known as viroplasms, where lipid droplets (LDs) interact with the viroplasm and facilitate viral replication. Despite the importance of LD formation in the life cycle of RVAs, the upstream molecules modulating LD formation remain unclear. This study aimed to find out the role of sterol regulatory element-binding proteins (SREBPs) in reprogramming of LD formation after RVA infection. Here, we demonstrate that RVA infection reprograms the sterol regulatory element-binding proteins (SREBPs)-dependent lipogenic pathways in virus-infected cells, and that both SREBP-1 and -2 transactivated genes, which are involved in fatty acid and cholesterol biosynthesis, are essential for LD formation. Our results showed that pharmacological inhibition of SREBPs using AM580 and betulin and inhibition of their downstream cholesterol biosynthesis (simvastatin for HMG-CoA reductase) and fatty acid enzymes (TOFA) negatively modulated the intracellular triacylglycerides and cholesterol levels and their resulting LD and viroplasm formations. Interestingly, pharmacological inhibition of SREBPs significantly reduced RVA protein synthesis, genome replication and progeny production. This study identified SREBPs-mediated lipogenic reprogramming in RVA-infected host cells, which facilitates virus replication through LD formation and its interaction with viroplasms, suggesting that SREBPs can be a potential target for the development of efficient and affordable therapeutics against RVA infection.
\end{abstract}

Key Words: Lipogenesis, Lipid droplet, Rotavirus, Sterol regulatory element-binding proteins, Viroplasm

\section{INTRODUCTION}

Species A rotaviruses (RVAs) are a major cause of severe diarrhea in children and a wide variety of young animals worldwide and lead to significant economic losses in the livestock industry (Dhama et al, 2009; Crawford and Desselberger, 2016; Tate et al, 2016). RVA messenger RNAs synthesized in and released from double-layer particles (DLPs) that escape from the endosome are used to translate viral proteins (Crawford et al, 2017). Newly assembled DLPs initiate a secondary wave of transcription and incorporation of the positive- sense RNA into replication intermediates (Ayala-Breton et al, 2009), and virus assembly occurs in specialized intracytoplasmic inclusions known as viroplasms (Silvestri et al, 2004). The NSP2 and NSP5 proteins are the major components of viroplasms and can form an empty viroplasm-like structure, although other viral proteins, such as VP1/2/3/6, and NSPG, may be required for this process in some strains (Fabbretti et al, 1999). After the emergence of viroplasms at 2 to $3 \mathrm{~h}$ post-infection (hpi), their number initially increases and then decreases over time (Eichwald et al, 2004; Carreño-Torres et al, 2010). The requirement of lipid droplets (LDs) for viroplasm 
formation in RVAs represents an example of how viruses hijack the cellular pathways for their own replication (Fabbretti et al, 1999; Eichwald et al, 2004; CarreñoTorres et al, 2010).

The biosynthesis of cholesterol, fatty acids, and triacylglycerides (TAGs), which are composed of fatty acids and glycerol through esterification, is tightly regulated by a family of transcription factors in mammals, known as sterol regulatory element-binding proteins (SREBPs) (Horton et al, 2002; Goldstein et al, 2006; Xiao and Song, 2013). Mammals contain two SREBPs, SREBP-1 and SREBP-2 (Horton et al, 2002; Goldstein et al, 2006; Xiao and Song, 2013). SREBP-1 produces two proteins, SREBP-1a and SREBP-1c, through alternative splicing (Horton et al, 2002; Goldstein et al, 2006; Xiao and Song, 2013). SREBP-1c signaling primarily regulates fatty acid biosynthesis genes, such as the fatty acid synthase (FAS) gene, whereas SREBP-2 predominantly transactivates cholesterol-related genes, such as HMGCoA reductase (HMGCR) and a low-density lipoprotein receptor (LDLR) genes (Horton et al, 2002; Goldstein et al, 2006; Xiao and Song, 2013). SREBP-1a targets both sides of genes (Horton et al, 2002; Goldstein et al, 2006; Xiao and Song, 2013). SREBPs are synthesized as inactive precursors bound to ER membranes (Horton et al, 2002; Goldstein et al, 2006; Xiao and Song, 2013). Low levels of cholesterol, oxysterols, and fatty acids modulate the SREBP pathway by binding to an SREBP cleavage-activating protein (cholesterol), insulin-induced genes (oxysterols), and Ubxd8 (unsaturated fatty acids) expressed on ER membranes, resulting in the ER-toGolgi trafficking of Scap-SREBP complexes, and the release and translocation of the transcriptionally active NH2-terminal domain (nSREBPs) into the nucleus (Horton et al, 2002; Goldstein et al, 2006; Xiao and Song, 2013). Activated SREBPs bind to the sterol regulatory element and then promote the transcription and expression of lipogenic genes (Horton et al, 2002; Goldstein et al, 2006; Xiao and Song, 2013).

Several RNA and DNA viruses are known to exploit SREBPs-dependent lipidomic reprogramming to facili- tate their replication (Park et al, 2009; Xiang et al, 2010; Olmstead et al, 2012; Qiao et al, 2013; Petersen et al, 2014; Kleinfelter et al, 2015; Hyrina et al, 2017; Wang et al, 2018; Wu et al, 2018; Casalino-Matsuda et al, 2019; Martin-Acebes et al, 2019; Meng et al, 2019; Yuan et al, 2019; Dias et al, 2020). However, the roles of SREBPs and their associated lipid biosynthesis pathways that modulate LD dynamics during RVA viroplasm formation have not been widely examined. Here, we demonstrated that the RVA-induced early activation of SREBP-1/2 determines the LD dynamics during RVA viroplasm formation by modulating their downstream fatty acid and cholesterol biosynthesis. Chemical inhibition suppresses RVA replication through negative modulation of LD formation and its major components, TAGs and cholesterol. Our findings highlight that SREBP-mediated fatty acid and cholesterol biosynthesis is necessary for LD formation and the facilitation of RVAs replication in the viroplasms, providing potential targets for the development of host-directed antiviral therapeutic agents against RVAs.

\section{MATERIALS AND METHODS}

\section{Cells and viruses}

Monkey kidney MA104 cells were purchased from the American Type Culture Collection (ATCC, Manassas, VA, USA) and grown in alpha minimal essential medium ( $\alpha$-MEM) or Dulbecco's modified Eagle's medium (Welgene, Daegu, South Korea), respectively, supplemented with 10\% fetal bovine serum (Gibco, Grand Island, NY, USA), $100 \mathrm{U} / \mathrm{mL}$ penicillin, and $100 \mu \mathrm{g} / \mathrm{mL}$ streptomycin (Lonza, Basel, Switzerland). The bovine RVA NCDV (G6P6[1]) strain (ATCC) were pre-activated with $10 \mu \mathrm{g} /$ $\mathrm{mL}$ crystalized trypsin (Cat. No. 27250-018, Gibco); the virus was propagated in MA104 and Caco-2 cells as described previously (Soliman et al, 2018). Virus titers were determined via the cell culture immunofluorescence assay using a monoclonal antibody (Mab) specific for RVA VP6 protein. Virus titers are expressed as fluorescence 
focus unit per milliliter (ffu/mL). The viral stocks were stored at $-80^{\circ} \mathrm{C}$ until analysis.

\section{Reagents and antibodies}

AM580, betulin, simvastatin, and TOFA were purchased from Cayman Chemical (Ann Arbor, MI, USA). AM580 and betulin were dissolved in dimethyl sulfoxide (DMSO), whereas simvastatin and TOFA were dissolved in RNase-free water to prepare stock solutions. SlowFade $^{\text {TM }}$ Gold Antifade Mountant with 4',6-diamidino2-phenylindole (DAPI) was purchased from Molecular Probes (Eugene, OR, USA). Mouse Mabs against FAS and HMGCR were obtained from BD Transduction Laboratories (Lexington, KY, USA). Mouse Mabs against glyceraldehyde 3-phosphate dehydrogenase (GAPDH) and $\alpha$-tubulin were obtained from Santa Cruz Biotechnology (Dallas, TX, USA) and Genetex (Irvine, CA, USA), respectively. The mouse Mab against RVA VP6 protein was purchased from Median Diagnostics (Chuncheon, South Korea), whereas hyperimmune serum raised in guinea pig against RVA non-structural protein-5 (NSP5) was kindly provided by Professor Oscar Burrone (International Center for Genetic Engineering and Biotechnology Padriciano, Trieste, Italy). Secondary antibodies included horseradish peroxidase-conjugated goat antirabbit IgG (Cell Signaling), horseradish peroxidase-conjugated goat anti-mouse IgG (Abcam), Alexa fluor (AF) 488-conjugated donkey anti-rabbit IgG, AF594-conjugated goat anti-guinea pig IgG, and AF594-conjugated goat anti-mouse IgG (Life Technologies).

\section{Cytotoxicity assay}

The cytotoxicity of the chemicals was assessed via 3-(4,5-dimethylthiazol-2-yl)-2,5-diphenyltetrazolium bromide (MTT) assay as described elsewhere (Soliman et al, 2018). Briefly, different concentrations of the desired chemicals, prepared in maintenance medium, were applied to a confluent monolayer of MA104 or Caco2 cells grown in 96-well plates. The plates were incubated for
$24 \mathrm{~h}$ at $37^{\circ} \mathrm{C}$ in a $\mathrm{CO}_{2}$ incubator. After removing the medium, $200 \mu \mathrm{L}$ of the MTT reagent was added to each well and the plates were further incubated for $2 \mathrm{~h}$ at $37^{\circ} \mathrm{C}$ in a $\mathrm{CO}_{2}$ incubator. Next, $150 \mu \mathrm{L}$ of DMSO was added to each well and the plate was incubated for $10 \mathrm{~min}$ at $20^{\circ} \mathrm{C}$. The absorbance was read at an optical density (OD) of $570 \mathrm{~nm}$ using an enzyme linked immunosorbent assay (ELISA) reader. Cell viability was measured using the following formula: $\left[\left(\mathrm{OD}_{\text {sample }}-\mathrm{OD}_{\text {blank }}\right) /\left(\mathrm{OD}_{\text {control }}-\mathrm{OD}_{\text {blank }}\right)\right] \times$ 100. The minimum concentration of each chemical that did not show cytotoxicity was used in this study.

\section{Treatment of cells with chemicals}

Confluent MA104 cells, grown in 6- or 12-well plates or 8-well chamber slides to the desired confluency, were washed twice with Dulbecco's PBS (DPBS; Welgene). The cells were divided into the following groups based on treatment with chemicals: mock-treated, pre-treated, post-treated, and pre/post-treated cells. In the pretreatment group, the cells were treated with the working concentration of chemicals for $1 \mathrm{~h}$ at $37^{\circ} \mathrm{C}$ before virus inoculation, whereas in the post-treatment group, the chemicals were added to the cells after viral adsorption and washing with DPBS. In the pre/post-treatment group, the cells were first incubated with the chemicals for $1 \mathrm{~h}$, washed twice with DPBS, and then inoculated with the virus. The cells were washed again with DPBS after viral adsorption and incubated with maintenance medium containing chemicals.

\section{Western blot analysis}

Western blot analysis was conducted to determine the expression levels of target proteins and viral proteins in culture cells as described elsewhere (Soliman et al, 2018). Immunoreactive bands were developed using an enhanced chemiluminescence reaction kit (DoGen, Seoul, South Korea), and images were obtained using the Davinch-K Western imaging system (Young Ltd., Kang-Nam, Seoul, South Korea). 
RT-qPCR

Total RNA in the cultured cells was quantified using RT-qPCR as described previously (Soliman et al, 2018; Yuan et al, 2019). The primer pairs are listed in Table 1. The threshold was automatically defined in the initial exponential phase, reflecting the cycle threshold (CT) values at the highest amplification rate. Relative amounts of RNA were calculated using the comparative
CT method, as previously described (Schmittgen and Livak, 2008).

\section{Immunofluorescence assay}

The immunofluorescence assay was performed to characterize the dynamics of LD formation and the colocalization of RVA protein NSP5, as described elsewhere (Soliman et al, 2018). The cells were ex-

Table 1. Primers used in this study

\begin{tabular}{|c|c|c|}
\hline Gene & Primer sequence & Size of amplicon \\
\hline \multirow[t]{2}{*}{$S R E B P-1 a$} & F: 5'-TCAGCGAGGCGGCTTTGGAGCAG-3' & 80 \\
\hline & R: 5'-CATGTCTTCGATGTCGGTCAG-3' & \\
\hline \multirow[t]{2}{*}{$S R E B P-1 c$} & F: 5'-GGAGGGGTAGGGCCAACGGCCT-3' & 80 \\
\hline & R: 5'-CATGTCTTCGAAAGTGCAATCC-3' & \\
\hline \multirow[t]{2}{*}{$S R E B P-2$} & F: 5'-GTCGGGTGTCATGGGCGGTG-3' & 105 \\
\hline & R: 5’-CTCGCCGCTGTCGTCGATCG-3' & \\
\hline \multirow[t]{2}{*}{ RVA VP6 } & F: 5'-TAGACCAAATAACGTTGAAGTTGA-3' & 237 \\
\hline & R: 5'-GATTCA CAAACTGCAGATTCAA-3' & \\
\hline \multirow[t]{2}{*}{ hGAPDH } & F: 5'-ATTCCACCCATGGCAAATTC-3' & 91 \\
\hline & R: 5'-CGCTCCTGGAAGATGGTGAT-3' & \\
\hline \multirow[t]{2}{*}{ mGAPDH } & F: 5'-AAGGTCATCCCAGAGCTGAA-3' & 138 \\
\hline & R: 5'-CTGCTTCACCACCTTCTTGA-3' & \\
\hline \multirow[t]{2}{*}{$A C C 1$} & F: 5'-GAGGGCTAGGTCTTTCTGGAAG-3' & 389 \\
\hline & R: 5'-CCACAGTGAAATCTCGTTGAGA-3' & \\
\hline \multirow[t]{2}{*}{ FAS } & F: 5'- CTTCCGAGATTCCATCCTACGC -3' & 131 \\
\hline & R: 5'- TGGCAGTCAGGCTCACAAACG -3' & \\
\hline \multirow[t]{2}{*}{$H M G C R$} & F: 5'-GGGAACCTCGGCCTAATGAA-3' & 156 \\
\hline & R: 5'-CACCACGCTCATGAGTTTCCA-3' & \\
\hline \multirow[t]{2}{*}{ DGAT1 } & F: 5'-GGCATCCTGAACTGGTGTGTG-3' & 366 \\
\hline & R: 5'-GAGCTTGAGGAAGAGGATGGTG-3' & \\
\hline \multirow[t]{2}{*}{ HMGCS1 } & F: 5'-GAGGGCTTCGTGGGACACATA-3' & 162 \\
\hline & R: 5'-GCCACTGGGCATGGATCTTT-3' & \\
\hline \multirow[t]{2}{*}{$M V K$} & F: 5'-CCTTGTGGCTGGCGTCAGAAA-3' & 191 \\
\hline & R: 5'-CGAGGGCATTCAGATGGTGCT-3' & \\
\hline \multirow[t]{2}{*}{$p M V K$} & F: 5'-GCCTTTCTCTCCGCGTGTCT-3' & 133 \\
\hline & R: 5'-GGAGCGGCAACAAGGAACA-3' & \\
\hline \multirow[t]{2}{*}{$S C D$} & F: 5'-TGCTGCCCACCTCTTCGGATAT-3' & 102 \\
\hline & R: 5'-TAGTTGTGGAAGCCCTCACCCA-3' & \\
\hline \multirow[t]{2}{*}{$L D L R$} & F: 5'-GCAGTGGGCGACAGATGTGAA-3' & 126 \\
\hline & R: 5'-GCACGTCTCCTGGGACTCATCA-3' & \\
\hline \multirow[t]{2}{*}{ RVA NSP3 } & F: 5'-GTTCGTTGTGCCTCATTCG-3' & 100 \\
\hline & R: 5'-TCGGAACGTACTTCTGGAC-3' & \\
\hline
\end{tabular}

$S R E B P$, sterol regulated element binding protein; RVA, species A rotavirus; hGAPDH, human glyceraldehyde 3-phosphate dehydrogenase; mGAPDH, mouse GAPDH; ACC, Acetyl-CoA carboxylase 1; FAS, fatty acid synthase; HMGCR, 3-hydroxy-3methylglutaryl-coenzyme A reductase; DGAT1, Diacylglycerol O-acyltransferase 1; $M V K$, mevalonate kinase; pMVK, phosphorylated MVK; $S C D$, stearoly-CoA desaturase; $L D L R$, low-density lipoprotein receptor. 
amined under an LSM 800 confocal microscope and analyzed via the Zeiss LSM image browser (Carl Zeiss, Oberkochen, Germany). The number and size of RVA viroplasms and LDs were measured, using the ImageJ program (National Institutes of Health, Bethesda, MD, USA), from 10 randomly taken images from each sample. The analysis was performed on approximately 100 cells, and the results were expressed as relative values. The standard error of difference in master values against which comparison was made was 5.804 . Virus titration in the cultured cells after chemical treatment was determined using an immunofluorescence assay as described previously (Soliman et al, 2018). The viral titer was expressed as $\mathrm{ffu} / \mathrm{mL}$.

\section{TAG and cholesterol colorimetric assays}

The intracellular levels of TAGs and cholesterol in the cultured cells were determined using a TAG colorimetric assay kit (Cayman Chemical) or cholesterol colorimetric assay kit (Abcam), respectively, according to the manufacturer's instructions. Briefly, MA104 cells were treated with chemicals and infected with the trypsin pre-activated NCDV strain (MOI=10), as described above. Untreated and non-transfected samples were used as the controls. The cultured cells $\left(1 \times 10^{6}\right.$ cells $)$ were counted using a hemocytometer after trypsinization and staining with methylene blue; next, the homogenized intestine tissue (3 mg) was prepared in each well of a 96-well plate and colorimetric assays were performed. The lysates were analyzed along with TAG or total cholesterol standard solutions prepared using the serial dilution method. Each value was measured at an OD of $570 \mathrm{~nm}$ using an ELISA reader and analyzed according to the manufacturer's instructions.

\section{Statistical analysis and software}

All experimental data, which were obtained in triplicate, were statistically analyzed using student's $t$-test or a one-way analysis of variance with GraphPad Prism version 8 software (GraphPad, Inc., La Jolla, CA, USA). $P$-values less than 0.05 were considered to indicate statistically significant results. Figures were generated using Adobe Photoshop CS6 and Prism 5 version 8.

\section{RESULTS}

\section{Dynamics of LD formation in RVA-infected MA104 cells}

We first examined the dynamics of LD formation in response to RVA infection. In MA104 cells infected with the bovine RVA strain NCDV (G6P6[1]) at a multiplicity of infection [MOI] of 10 fluorescence focus units per cell (ffu/cell), the number of dots (viroplasms) stained by the antibody against the RVA NSP5 protein, a major viral protein of RVA-induced viroplasms (Fabbretti et al, 1999), increased for up to $6 \mathrm{~h}$ hpi and decreased thereafter (Fig. 1A, 1B). These results are consistent with those of the previous studies regarding the dynamics of the numbers and sizes of viroplasms induced by both simian SA11 and RRV and porcine OSU strains (Eichwald et al, 2004; Carreño-Torres et al, 2010). However, the number of LDs stained by BODIPY gradually increased for up to $6 \mathrm{hpi}$ and then rapidly decreased under the same conditions (Fig. 1A), and the LD sizes appeared to be comparatively larger at $12 \mathrm{hpi}$ (Fig. 1A). As expected, LDs usually colocalized with the NSP5 protein for up to 6 hpi (Fig. 1A).

Because the main components of LDs are TAGs and cholesterol esters (Herker and Ott, 2012), the intracytoplasmic dynamics of TAGs and cholesterol may be similar to those in RVA-induced LDs. As the dynamics of the LD number and size in response to RVA infection appeared within $12 \mathrm{hpi}$, we next examined the dynamics of intracellular TAG and cholesterol levels, using TAG and cholesterol colorimetric assays, for up to $10 \mathrm{hpi}$ in RVA-infected MA104 cells. Compared to those in mockinfected cells, the TAG and cholesterol concentrations gradually increased, peaked at 4 hpi (an approximately a 4-fold increase) or $6 \mathrm{hpi}$ (an approximately a 4-fold increase), respectively, and then decreased over time 

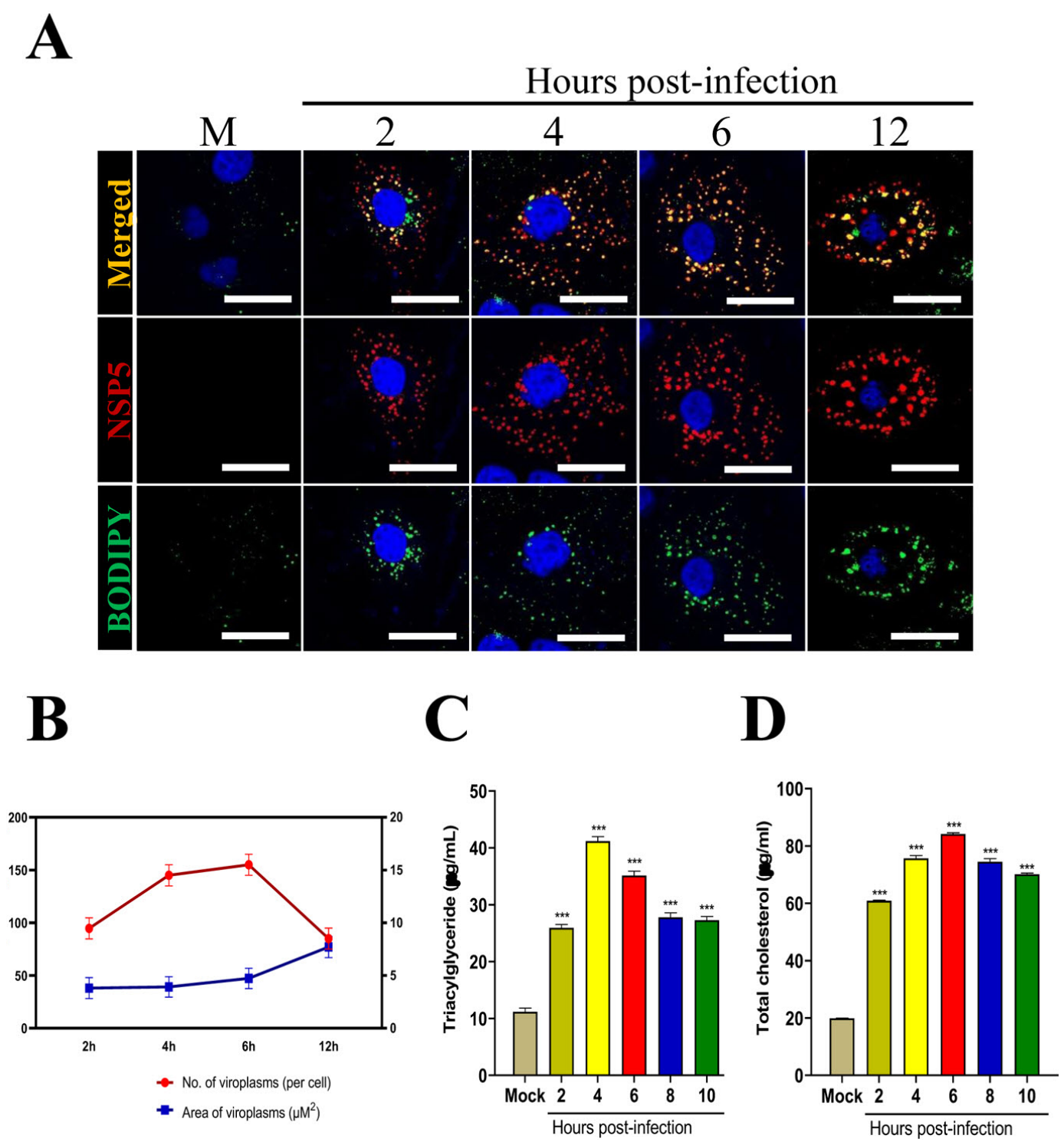

Fig. 1. Time course analysis of viroplasm and lipid droplet (LD) formation following rotavirus infection. MA104 cells were infected with the bovine rotavirus strain $\mathrm{NCDV}$ (MOI=10 ffu/cell) for the indicated time periods. (A) After fixation and permeabilization, the cells were prepared for immunofluorescence assay using hyperimmune guinea pig sera against NSP5 to visualize viroplasms, and BODIPY to detect LDs. (B) Number and size of viroplasms were determined using the ImageJ software. (C, D) Cell lysates were processed for colorimetric quantification assays to detect the intracellular concentrations of triacylglycerides (C) and cholesterol (D). Scale bars in panel A correspond to $10 \mu \mathrm{m}$. All experiments were performed in triplicate. Data in panels B $\sim \mathrm{D}$ represent the mean \pm standard deviation of values obtained from three independent experiments. Differences were evaluated using a one-way ANOVA. ${ }^{*} P<0.05 ;{ }^{*} P<0.01$; $* * * P<0.001$.

in virus-infected cells (Fig. 1C, 1D). Collectively, RVAs showed distinct dynamics for LDs and their major components, TAGs and cholesterol, in association with RVA replication, with an early increase and later decrease in RVA-infected cells.

\section{RVA-induced early activation of SREBP-mediated lipogenic reprogramming in the monkey kidney epithelial cells}

SREBP-1 and - 2 are key transcription factors for cholesterol and fatty acid biosynthesis (Horton et al, 2002; 
Goldstein et al, 2006; Xiao and Song, 2013). Deficiencies of intracellular cholesterol and fatty acids results in the cleavage of both SREBPs into NH2-terminal and $\mathrm{COOH}-$ terminal domains, leading to translocation of the transcriptionally active NH2-terminal domain (nSREBP) into the nucleus to transactivate lipogenic genes (Horton et al, 2002; Goldstein et al, 2006; Xiao and Song, 2013). Nevertheless, SREBPs may modulate the RVA-induced early formation of LDs and their major components, TAGs and cholesterol. Therefore, we determined the transcription levels of both SREBP-1 and -2 in RVAinfected cells. As shown in Fig. 2A, RVA infection in MA104 cells induced a significantly early elevation of the mRNA levels of SREBP-1a (1.4 \pm 0.5 fold-, $1.7 \pm 0.5$ fold-, and $1.5 \pm 0.5$-fold at 2,4 , and 6 hpi, respectively), SREBP-1C (1.5 $\pm 0.5-, 1.5 \pm 0.5-$, and $1.3 \pm 0.5$-fold at 2 , 4, and $6 \mathrm{hpi}$, respectively), and SREBP-2 (1.3 $\pm 0.5-, 1.6 \pm$ $0.5-$, and $1.4 \pm 0.5$-fold at 2,4 , and $6 \mathrm{hpi}$, respectively). Additionally, the mRNA levels of their downstream lipogenic genes in RVA-infected cells gradually increased and reached a peak at different times depending on the genes; a $2.8 \pm 0.5$-fold increase in ACC level at $6 \mathrm{hpi}, 2.1 \pm 0.5-$ fold increase in FAS level at $4 \mathrm{hpi}, 2.5 \pm 0.5$-fold increase in HMGCR level at $6 \mathrm{hpi}, 2.1 \pm 0.5$-fold increase in stearoyl-CoA desaturase $(S C D)$ level at $6 \mathrm{hpi}, 1.8 \pm 0.5$-fold increase in diacylglycerol O-acyltransferase 1 (DGAT1) level at $6 \mathrm{hpi}, 2.4 \pm 0.5$-fold increase in 3-hydroxy-3methylglutaryl-CoA synthase 1 (HMGCS1) level at $8 \mathrm{hpi}$, $2.0 \pm 0.5$-fold increase in $L D L R$ level at $6 \mathrm{hpi} 1,1.6 \pm$ 0.5 -fold increase in mevalonate kinase $(M V K)$ level at 6 hpi, and 1.8 \pm 0.5 -fold increase in phosphorylated MVK ( $p M V K)$ level at 6 hpi (Fig. 2B). These results suggest that RVA infection induces early activation of SREBPsmediated fatty acid and cholesterol biosynthesis for LD formation and early inhibition of host catabolism.

\section{Chemical inhibition of SREBPs reduces RVA replication and LD formation}

To further investigate the roles of SREBP-1 and -2 in RVA replication and LD formation, MA104 cells were treated with non-toxic doses of AM580 and betulin before and after the virus adsorption step (pre/posttreatment); untreated cells were used as the controls (Figs. 3A, 3B, 4). Treatment of MA104 cells with AM580 or betulin either individually or in combination, significantly reduced the expression levels of the viral VP6 protein (Fig. 4A), the total viral RNA load (an approximately 100,000-fold reduction after treatment with both AM580 and betulin, Fig. 4B), and viral progeny produc-
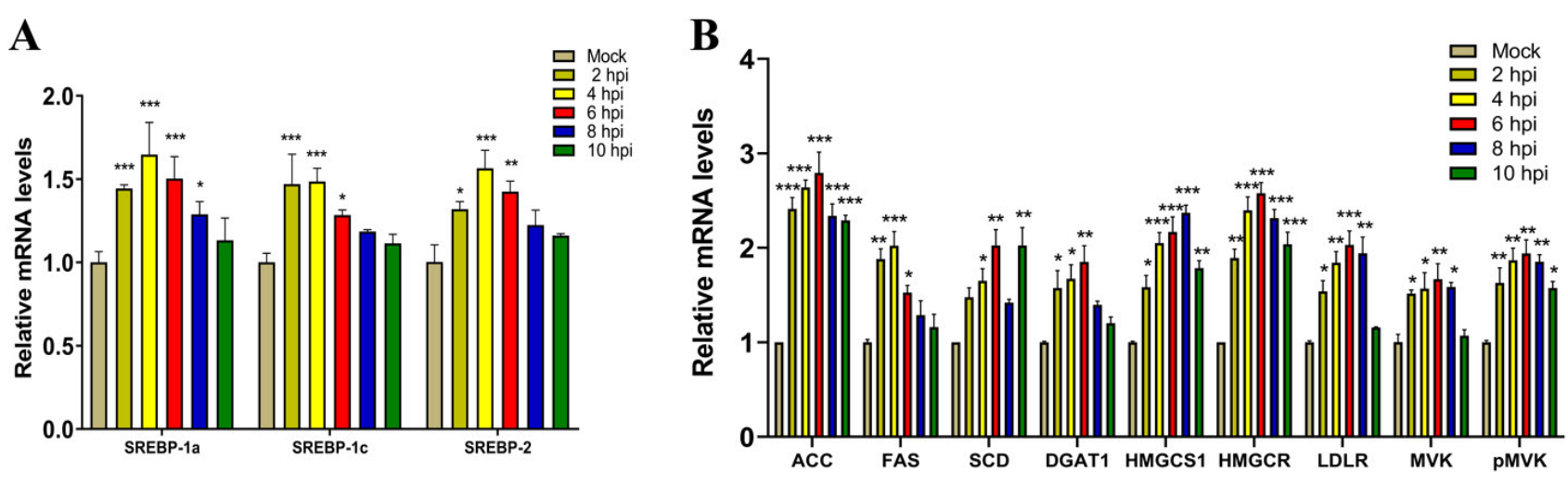

Fig. 2. Rotavirus-induced $S R E B P$-mediated lipogenic reprogramming in rotavirus-infected cells. MA104 cells were infected with the bovine rotavirus NCDV (MOI=10 ffu/cell) strain for the indicated time periods. (A, B) Relative mRNA expression of SREBPs $(S R E B P$ $1 a,-1 c$, and -2) (A) and SREBP-associated lipogenic enzyme genes including acetyl-CoA carboxylase (ACC), fatty acid synthase (FAS), stearoyl CoA desaturase $(S C D)$, diacylglycerol $O$-acyltransferase 1 (DGAT1), 3-hydroxy-3-methylglutaryl-CoA synthase 1 (HMGCS1), HMG-CoA reductase (HMGCR), low-density lipoprotein receptor ( $L D L R)$, mevalonate kinase (MVK), and phosphorylated MVK $(p M V K)(B)$ was determined with RT-qPCR. All experiments were performed in triplicate. Differences were evaluated using a one-way ANOVA. $* P<0.05 ; * * P<0.01 ; * * * P<0.001$. 
A

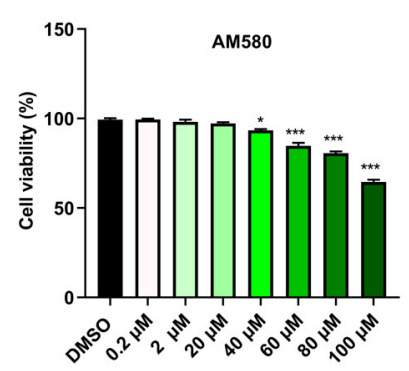

B

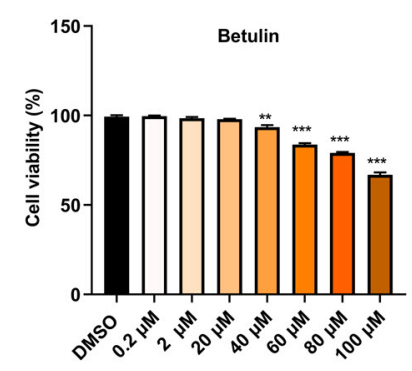

C

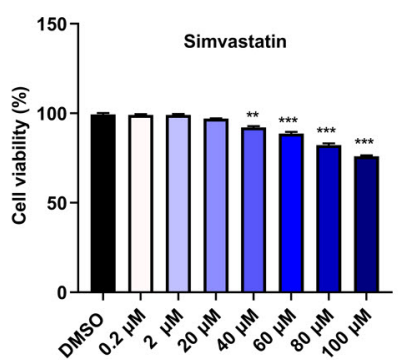

D

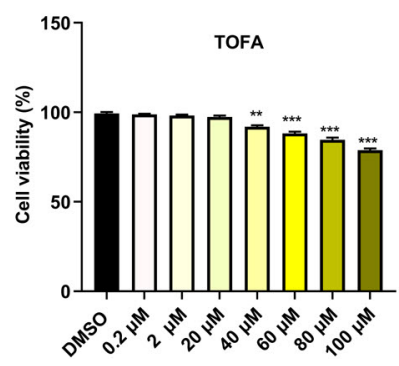

Fig. 3. Determination of the cytotoxicity of chemicals via the MTT assay. (A D) MA104 cells, grown in a 96-well plate, were incubated with various concentrations of the indicated chemicals in triplicate for $24 \mathrm{~h}$ at $37^{\circ} \mathrm{C}$. The chemical-containing media were thoroughly removed and replaced with $200 \mu \mathrm{L}$ of the MTT solution, followed by incubation for $4 \mathrm{~h}$ at $37^{\circ} \mathrm{C}$. Each well was incubated with $150 \mu \mathrm{L}$ of DMSO for $10 \mathrm{~min}$ at $20^{\circ} \mathrm{C}$. Cell viability was measured using an ELISA reader at an OD value of $570 \mathrm{~nm}$.

tion (a 3-log10 reduction after treatment with AM580 and betulin, Fig. 4C). Furthermore, treatments with AM580 or betulin markedly inhibited the expression of the SREBP-associated lipogenic enzymes HMGCR and FAS (Fig. 4A), and the intracellular levels of TAGs (Fig. 4D) and cholesterol (Fig. 4E). Taken together, these results demonstrate that RVA infection reprograms SREBPs-mediated lipogenic pathways in host cells.

\section{Chemical inhibition of SREBPs-associated lipogenic enzymes reduces RVA replication and LD formation}

As RVA induced lipogenic enzymes via SREBPs, we further evaluated whether chemicals inhibiting the enzymes involved in cholesterol and fatty acid biosynthesis affect RVA replication and LD formation. MA104

cells were pretreated, post-treated, or pre- and posttreated with different concentrations of either the ACC inhibitor 5-(tetradecyloxy)-2-furoic acid (TOFA) or the HMGCR inhibitor simvastatin and infected with the RVA NCDV strain; untreated cells were used as the controls. Treatment with TOFA and simvastatin, the maximum concentrations of which were non-toxic (Fig. 3C, 3D), significantly reduced viral VP6 protein expression (Fig. 5A, 5B), the total viral RNA load (an approximately 1000-fold decrease after treatment with TOFA and simvastatin, Fig. 5C), and viral progeny production (approximately 2.5-log10 reduction by treatment with TOFA and simvastatin, Fig. 5D). Moreover, treatment with TOFA and simvastatin induced significant reduction in TAG and cholesterol levels in the RVA-infected cells (Fig. 5E, 5F). Inhibition of RVA replication through downregulation of fatty acid synthesis by treatment with TOFA is consistent with previously reported results (52). Taken together, these results demonstrate that RVA infection reprograms SREBPs-mediated lipogenic pathways in host cells.

\section{DISCUSSION}

RVA-induced LDs interact with viroplasms, where viral RNA replication and DLP morphogenesis occur (Eichwald et al, 2004; Carreño-Torres et al, 2010); this suggests that cellular lipid biogenesis is essential for RVA-induced viroplasm formation followed by RVA replication (Cheung et al, 2010; Gaunt et al, 2013). However, the dynamics of transcription factor(s) and their lipogenic genes for lipid biosynthesis involved in LD formation and RVA replication have not been explored in detail. Here, we demonstrated that RVA infection induces SREBP-mediated lipogenic reprogramming in host cells, leading to early formation of LDs and their interactions with viroplasms, thereby facilitating virus replication. Our data revealed that a basic SREBP-mediated lipogenic transactivation event occurred during RVA-induced LD formation, which may facilitate the development of antiviral therapies against RVA infec- 
A

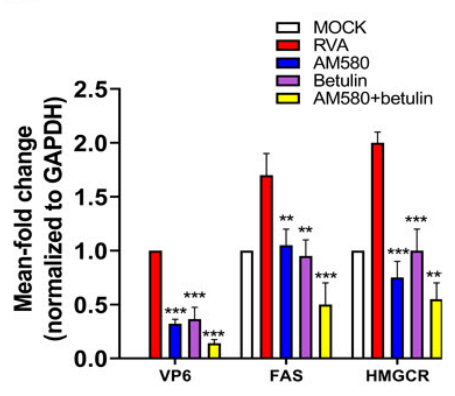

D

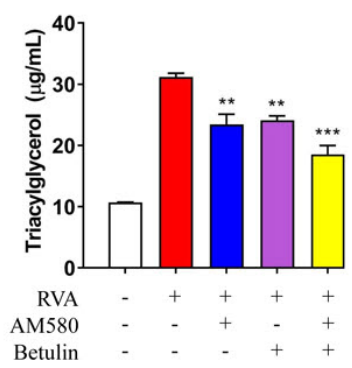

B

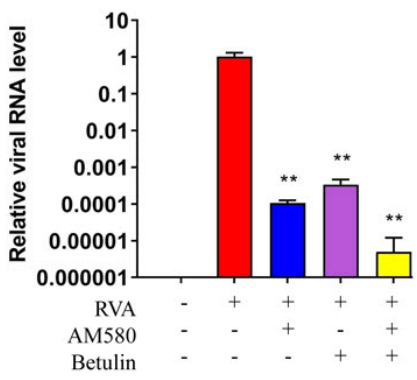

E

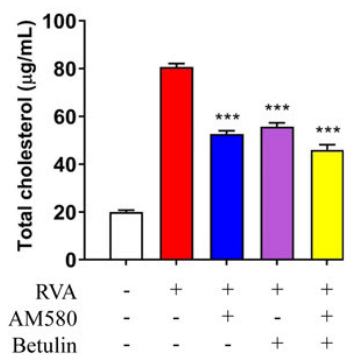

C

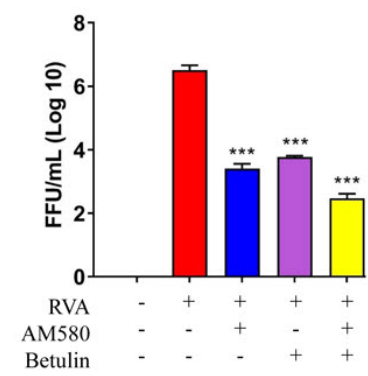

Fig. 4. SREBP inhibition suppressed rotavirus replication via blocking LD formation. (A $\sim$ E) MA104 cells were treated with the SREBP inhibitors AM580 and/or betulin prior to and after inoculation with the rotavirus NCDV strain (MOI=10 ffu/cell). (A) Cells were harvested at $12 \mathrm{~h}$ post-infection and processed for western blot analysis to determine the expression levels of VP6, FAS, and HMGCR. GAPDH was used as the loading control. Intensities of target proteins relative to those of GAPDH were determined using densitometric analysis. (B) Viral genome copy numbers in the cell lysates under the above-mentioned experimental conditions were determined via RT-qPCR, and relative viral RNA levels in the cells treated with AM580 and/or betulin were compared with those in mock-treated and virus-infected cells. (C) Viral titers were determined using a cell culture immunofluorescence assay with cell lysates produced by three cycles of freezing and thawing; the results are expressed in fluorescent focus forming unit per milliliter (ffu/mL). (D, E) Cell lysates were processed for colorimetric quantification assays to detect the intracellular concentrations of triglycerides (D) and cholesterol (E). All experiments were performed in triplicate. The data in panels A $\sim \mathrm{E}$ represent the mean \pm standard deviation of values obtained from three independent experiments. Differences were evaluated using a one-way ANOVA. $* * P<0.01 ; * * * P<0.001$.

tions.

In the present study, the bovine NCDV strain induced an early increase and a late decrease in the number of viroplasms and a gradual increase in their size. These dynamics of viroplasms are in accordance with those induced by other RVA strains, such as the rhesus RVA strains SA11 and RRV and porcine strain OSU (Eichwald et al, 2004; Carreño-Torres et al, 2010). Interestingly, in parallel with the dynamics of LD numbers, RVA infection induced similar dynamics for both SREBP-1 and -2 and the associated lipogenic enzymes at the mRNA levels. Early upregulation and late downregulation of SREBP-mediated lipogenic pathways eventually influenced the intracellular concentrations of TAGs and cholesterol, which are major components of LDs. Chemical inhibition markedly reduced the formation of both viroplasms and LDs via blockade of lipogenic enzymes, such as FAS and HMGCR. A recent study reported that the multivalent Ser/Glu-rich protein NSP5 and the viral RNA chaperone NSP2 undergo liquid-liquid phase separation and form RNA-rich liquid condensates in the early stage of RVA infection (Geiger et al, 2020). However, these liquid states of RNA-protein condensates turned into solid state at the late stage of RVA infection (Geiger et al, 2020). This is partially consistent with our results showing early upregulation and late downregulation of SREBP-mediated lipogenic pathways. Collectively, our data demonstrated that RVA infection 
A

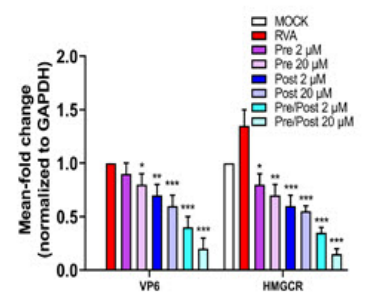

B

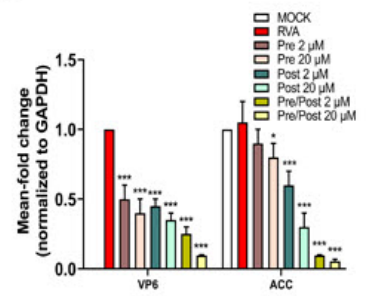

D

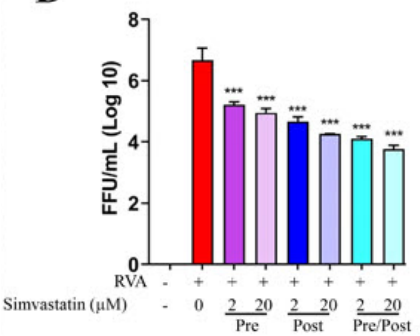

C
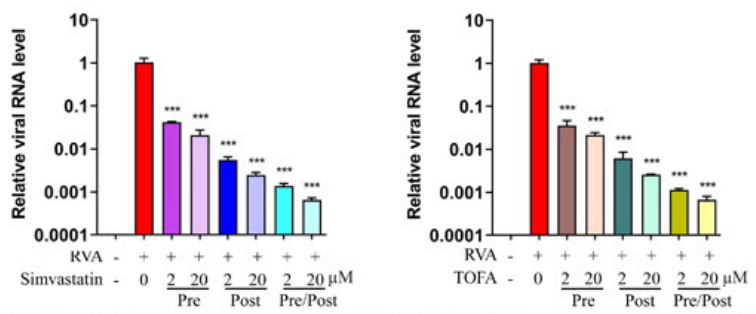

E

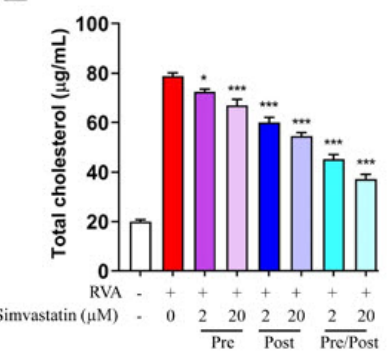

F

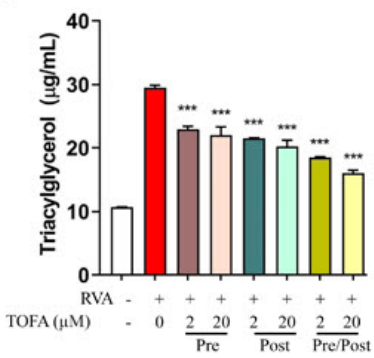

Fig. 5. Chemical inhibition of lipogenic enzymes with simvastatin and TOFA suppressed rotavirus replication by blocking LD formation. (A, B) MA104 cells were treated with an HMGCR inhibitor (simvastatin) or ACC inhibitor (TOFA) either prior to inoculation with the rotavirus strain NCDV (MOI=10 ffu/cell) (Pre), after inoculation (Post), or prior to and after inoculation (Pre/post). Cells were harvested $12 \mathrm{~h}$ post-infection and processed for western blot analysis to determine the expression levels of VP6, HMGCR, and ACC. GAPDH was used as the loading control. Intensities of target proteins relative to those of GAPDH were determined using densitometric analysis. $(\mathrm{C} \sim \mathrm{F})$ MA104 cells were treated with an HMGCR inhibitor (simvastatin) or ACC inhibitor (TOFA) prior to and after inoculation with the rotavirus strain NCDV (MOI=10 ffu/cell). (C) Viral genome copy numbers in cell lysates under the above-mentioned experimental conditions were determined using RT-qPCR, and relative viral RNA levels in the cells treated with simvastatin (left graph) or TOFA (right graph) were compared with those in mock-treated and virus-infected cells. (D) Viral titers were determined using a cell culture immunofluorescence assay with cell lysates produced by three cycles of freezing and thawing; the results are expressed in fluorescent focus forming unit per milliliter (ffu/mL). (E, F) Cell lysates were processed for colorimetric quantification assays to detect the intracellular concentrations of cholesterol (E) and triglycerides (F). All experiments were performed in triplicate. The data in panels $A \sim F$ represent the mean \pm standard deviation of values obtained from three independent experiments. Differences were evaluated using a one-way ANOVA. $* P<0.05 ; * * P<0.01 ; * * * P<0.001$.

induced early activation of SREBP-mediated lipogenic reprogramming in host cells to form LDs as the facilitators of RVA viroplasms.

AM580 and betulin used in this study specifically interfere with the SREBP pathway and reportedly have broad-spectrum antiviral activities (Yuan et al, 2019). However, their anti-RVA activity has not been reported to date. Chemical inhibition of SREBPs in RVA-infected cells led to decreased fatty acid and cholesterol biosynthesis as well as decreased viral progeny production. Additionally, we found that direct inhibition of the SREBP downstream regulators HMGCR and ACC via simvastatin and TOFA not only decreased total lipid contents or LD homeostasis but also suppressed RVA replication. Given the dependence of RVA replication on host lipid metabolism and that host cellular factors are often less susceptible to mutation compared to viral elements (Zhang et al, 2017), SREBP-mediated lipogenic pathways are potential targets for the development of drugs against RVA infection. Future pharmacokinetic and clinical studies of these inhibitors are needed to develop novel treatment strategies for patients with RVA-induced severe acute diarrhea.

In conclusion, we observed SREBP-mediated lipogenic reprogramming in RVA-infected cells. RVAs utilize the SREBP-1 and SREBP-2 transcription factors to transactivate genes involved in fatty acid and cholesterol biosynthesis for the formation of LDs as facilitators of viroplasms, where RVA replication and assembly occur. Compounds intrusive to the SREBP-mediated lipogenic 
pathways not only reduced fatty acid and cholesterol biosynthesis but also suppressed viral replication. Therefore, pharmacological inhibitors against SREBPs may have clinical applicability for treating patients with RVA-induced diarrhea.

\section{ACKNOWLEDGEMENTS}

SIP and KOC were supported by grant (2019R1A2C 1006502) from the Basic Science Research Program through the National Research Foundation of Korea, which is funded by the Ministry of Science, ICT and Future Planning, Republic of Korea.

\section{CONFLICT OF INTEREST}

No potential conflict of interest relevant to this article was reported.

\section{ORCID}

Ahsan Naveed, https://orcid.org/0000-0001-9378-5086 Yeong-Bin Baek, https://orcid.org/0000-0003-1703-7742 Mahmoud Soliman, https://orcid.org/0000-0003-4110-3703 Muhammad Sharif, https://orcid.org/0000-0002-8500-3862 Sang-Ik Park, https://orcid.org/0000-0003-1709-0324 Mun-Il Kang, https://orcid.org/0000-0003-1595-1717

\section{REFERENCES}

Ayala-Breton C, Arias M, Espinosa R, Romero P, Arias CF, López S. 2009. Analysis of the kinetics of transcription and replication of the rotavirus genome by RNA interference. J Virol. 83(17): 8819-8831.

Carreño-Torres JJ, Gutiérrez M, Arias CF, López S, Isa P. 2010. Characterization of viroplasm formation during the early stages of rotavirus infection. Virol J 7: 350 .

Casalino-Matsuda M, Chen F, Matsuda A, Sporn PHS. 2019. Hypercapnia increases influenza A virus replication in airway epithelium through a sterol- regulatory element binding protein 2 (SREBP2)dependent mechanism. Am J Respir Crit Care Med 199: A5748.

Cheung W, Gill M, Esposito A, Kaminski CF, Courousse N, Chwetzoff S, Trugnan G, Keshavan N, Lever A, Desselberger U. 2010. Rotaviruses associate with cellular lipid droplet components to replicate in viroplasms, and compounds disrupting or blocking lipid droplets inhibit viroplasm formation and viral replication. J Virol 84: 6782-6798.

Crawford SE, Desselberger U. 2016. Lipid droplets form complexes with viroplasms and are crucial for rotavirus replication. Curr Opin Virol 19: 11-15.

Crawford SE, Ramani S, Tate JE, Parashar UD, Svensson L, Hagbom M, Franco MA, Greenberg HB, O’Ryan M, Kang G, Desselberger U, Estes MK. 2017. Rotavirus infection. Nat Rev Dis Primers 3: 17083.

Dhama K, Chauhan RS, Mahendran M, Malik SV. 2009. Rotavirus diarrhea in bovines and other domestic animals. Vet Res Commun 33: 1-23.

Dias SSG, Soares VC, Ferreira AC, Sacramento CQ, Fintelman-Rodrigues N, Temerozo JR, Teixeira L, da Silva AN, Barreto E, Mattos M, de Freitas CD, Azevedo-Quintanilha IG, Manso PP, Miranda MD, Siqueira MM, Hottz ED, Pão CRR, Bou-Habib DC, Brarreto-Vieira DF, Bozza FA, Souza TM, Bozza PT. 2020. Lipid droplets fuel SARS-CoV-2 replication and production of inflammatory mediators. PLoS Pathog 16: e1009127.

Eichwald C, Rodriguez JF, Burrone OR. 2004. Characterization of rotavirus NSP2/NSP5 interactions and the dynamics of viroplasm formation. J Gen Virol 85: 625-634.

Fabbretti E, Afrikanova I, Vascotto F, Burrone OR. 1999. Two non-structural rotavirus proteins, NSP2 and NSP5, form viroplasm-like structures in vivo. J Gen Virol 80 (Pt 2): 333-339.

Gaunt ER, Zhang Q, Cheung W, Wakelam MJO, Lever AML, Desselberger U. 2013. Lipidome analysis of rotavirus-infected cells confirms the close interaction of lipid droplets with viroplasms. J GenVirol 
94: $1576-1586$.

Geiger F, Acker J, Papa G, Wang X, Arter WE, Saar KL, et al. Liquid-liquid phase separation underpins the formation of replication factories in rotaviruses. EMBO J 2021; e107711.

Goldstein JL, DeBose-Boyd RA, Brown MS. 2006. Protein sensors for membrane sterols. Cell 124: 35-46.

Herker E, Ott M. 2012. Emerging role of lipid droplets in host/pathogen interactions. J Biol Chem 287: 2280-2287.

Horton JD, Goldstein JL, Brown MS. 2002. SREBPs: activators of the complete program of cholesterol and fatty acid synthesis in the liver. J Clin Invest 109: 1125-1131.

Hyrina A, Meng F, McArthur SJ, Eivemark S, Nabi IR, Jean F. 2017. Human subtilisin kexin isozyme-1 (SKI-1)/site-1 protease (S1P) regulates cytoplasmic lipid droplet abundance: A potential target for indirect-acting anti-dengue virus agents. PLoS One 12: e0174483.

Kleinfelter LM, Jangra RK, Jae LT, Herbert AS, Mittler E, Stiles KM, Wirchnianski AS, Kielian M, Brummelkamp TR, Dye JM, Chandran K. 2015. Haploid genetic screen reveals a profound and direct dependence on cholesterol for hantavirus membrane fusion. mBio 6: e00801.

Martin-Acebes MA, de Oya NJ, Saiz JC. 2019. Lipid metabolism as a source of druggable targets for antiviral discovery against Zika and other flaviviruses. Pharmaceuticals (Basel) 12: 97.

Meng Z, Liu Q, Sun F, Qiao L. 2019. Hepatitis C virus nonstructural protein $5 \mathrm{~A}$ perturbs lipid metabolism by modulating AMPK/SREBP-1c signaling. Lipids Health Dis 18: 191.

Olmstead AD, Knecht W, Lazarov I, Dixit SB, Jean F. 2012. Human subtilase SKI-1/S1P is a master regulator of the $\mathrm{HCV}$ lifecycle and a potential host cell target for developing indirect-acting antiviral agents. PLoS Pathog 8: e1002468.

Park CY, Jun HJ, Wakita T, Cheong JH, Hwang SB. 2009. Hepatitis $\mathrm{C}$ virus nonstructural $4 \mathrm{~B}$ protein modu- lates sterol regulatory element-binding protein signaling via the AKT pathway. J Biol Chem 284: 9237-9246.

Petersen J, Drake MJ, Bruce EA, Riblett AM, Didigu CA, Wilen CB, Malani N, Male F, Lee FH, Bushman FD, Cherry S, Doms RW, Bates P, Briley K, Jr. 2014. The major cellular sterol regulatory pathway is required for Andes virus infection. PLoS Pathog 10: e1003911.

Qiao L, Wu Q, Lu X, Zhou Y, Fernandez-Alvarez A, Ye L, Zhang X, Han J, Casado M, Liu Q. 2013. SREBP1a activation by HBx and the effect on hepatitis B virus enhancer II/core promoter. Biochem Biophys Res Commun 432: 643-649.

Schmittgen TD, Livak KJ. 2008. Analyzing real-time PCR data by the comparative C(T) method. Nat Protoc 3(6): 1101-8.

Silvestri LS, Taraporewala ZF, Patton JT. 2004. Rotavirus replication: plus-sense templates for doublestranded RNA synthesis are made in viroplasms. J Virol 78: 7763-7774.

Soliman M, Cho EH, Park JG, Kim JY, Alfajaro MM, Baek YB, Kim DS, Kang MI, Park SI, Cho KO. 2018. Rotavirus-induced early activationof the RhoA/ ROCK/MLC signaling pathway mediates the disruption of tight junctions in polarized MDCK Cells. Sci Rep 8: 13931.

Tate JE, Burton AH, Boschi-Pinto C, Parashar UD, World Health Organization-Coordinated Global Rotavirus Surveillance N. 2016. Global, regional, and national estimates of rotavirus mortality in children $<5$ years of age, 2000-2013. Clin Infect Dis 62 Suppl 2: S96-S105.

Wang L, Xie W, Zhang L, Li D, Yu H, Xiong J, Peng J, Qiu J, Sheng H, He X, Zhang K. 2018. CVB3 nonstructural 2A proteinmodulates SREBP1a signaling via the MEK/ERK pathway. J Virol 92: e01060-18.

Wu Q, Li Z, Liu Q. 2018. An important role of SREBP-1 in $\mathrm{HBV}$ and $\mathrm{HCV}$ co-replication inhibition by PTEN. Virology 520: 94-102.

Xiang Z, Qiao L, Zhou Y, Babiuk LA, Liu Q. 2010. Hepa- 
titis $\mathrm{C}$ virus nonstructural protein-5A activates sterol regulatory element-binding protein-1c through transcription factor Sp1. Biochem Biophys Res Commun 402: 549-553.

Xiao X, Song BL. 2013. SREBP: a novel therapeutic target. Acta Biochim Biophys Sin (Shanghai) 45: 2-10.

Yuan S, Chu H, Chan JF, Ye ZW, Wen L, Yan B, Lai PM, Tee KM, Huang J, Chen D, Li C, Zhao X, Yang D, Chiu MC, Yip C, Poon VK, Chan CC, Sze KH, Zhou
J, Chan IH, Kok KH, To KK, Kao RY, Lau JY, Jin DY, Perlman S, Yuen KY. 2019. SREBP-dependent lipidomic reprogramming as a broad-spectrum antiviral target. Nat Commun 10: 120.

Zhang J, Lan Y, Sanyal S. 2017. Modulation of lipid droplet metabolism-A potential target for therapeutic intervention in flaviviridae infections. Front Microbiol 8: 2286. 